\title{
An iteration method for predicting vehicular trajectory based on car-following models
}

\author{
Fei Teng ${ }^{1, a}$, Jianqun Wang ${ }^{2, b^{*}}$ and Rui Chai ${ }^{3, c}$ \\ 1, 2, 3 School of Mechanical Engineering, Beijing Institute of Technology \\ 5 South Zhongguancun, Beijing 100081, China \\ a2120130506@bit.edu.cn, bwangjq@bit.edu.cn, cchairui20@126.com
}

\section{Keywords: Car-following models; Trajectory Prediction; Collision Warning System.}

Abstract. In the recent two decades, the improvement of vehicular network gave momentum to the research of vehicle collision warning systems, which focus on curbing wounded and fatalities in crashes, increase the requirement to uncover the mechanism of driving. Car-following algorithm, a series of methodology using formula to express the interaction between drivers and vehicles, have reproduce several intricate phenomenon. In this paper, some highly-cited car-following algorithm are calibrated based on the positional information in real traffic and exploited to predict future trend of vehicles more precisely. By analyzing the error distribution in prediction vehicles' trajectory for each calibrated car-following models, an iteration method is used to mitigation positional errors and thus may help to resolve the errors caused by anticipation or stochastic characteristic of drivers. The results indicate optimistic performance of proposed trajectory prediction model especially in constant car-following situation.

\section{Introduction}

Current research trend in traffic safety promote improving active vehicle safety technology, which may be a primary reason of conspicuously reduction of both people injured and fatalities in traffic accidents in recent years (an overview of crashes published in Feb 2015 by National Highway Traffic Safety Administration indicate that in 2013 deaths of passenger car occupants decrease 38\% comparing 2004 [1]). Many vehicular forward collision warning systems and related applications, such as systems integrating vehicular network (vehicle-to-vehicle or vehicle-to-infrastructure communication) [2, 3], have demonstrated their potential in connection and reliability to preventing collision.

Given more accurate and diverse information helping to predict crashes, however, ignorance of collision warning algorithm will dilute the advantage of the informations: collision warning algorithm require to scrutinize drivers' characteristics based on the history information gleaned from sensors in car. Atif Mehmood claimed in his dissertation that reaction time, as well as many other driver characteristics, have critical influence in forecast future trend of vehicles [4]. In the scenario of car-following situation, many car-following models such as Atif Mehmood's reaction time based model in his thesis, have potential to alleviate the difficulty in charactering driving characteristics and corresponding exploitation in collision prediction.

On the other hand, car-following models, a bench of transportation research begin around 1950's, have demonstrated capability in reproducing special traffic phenomenon in both macroscopic and microscopic situation. Denos C. Gazis et al. [5] established relevancy between macroscopic traffic flow theory and microscopic vehicle dynamic by using mathematical reasoning, the interaction between vehicles were explained by stimulus-response equation for each driver - that is, each driver response the dynamic attribute changes of leading vehicle by adjust acceleration of self-vehicle according to specific sensitivity character - to understand the flow-density relation from an individual vehicle's view point. Martin Treiber also shed light on the mechanism of traffic congestion by using their intelligent driver model [6], to reproduce specific congestion scenario using flow data recorded in real traffic. Besides, asymmetry acceleration/deceleration, hysteresis, anticipation and relaxation has also explained and expressed by specific car-following models, respectively. 
In this paper, we review some findings about car-following models for predicting vehicles' trajectories in Chapter II. Then we introduce a framework of iteration method which serve to mitigation positional errors in trajectory prediction by calibrated car-following models in Section 3.1, then parameter calibration and model examination is described in other sections in Chapter III. Eventually, conclusions and future work are drawn in Chapter IV.

\section{Overview of car-following models}

There are several methodologies for express traffic dynamic mathematically, basically in two interrelated prospective: on one hand, macroscopic models explain the relation among statistical quantities such as density, average speed, flow rate, and lane-changing probability, and on the other hand, microscopic ones discuss the motion of individual vehicles. To predict the vehicles' trajectories better, we analyzed highly-cited thesis about car-following strategy (that is, microscopic traffic models) and select a batch among them which have developed mathematic models to reproduce complex traffic phenomenon or empirical findings with mere three or less parameters (plethoric parameter require more data).

The most well-known model, Gazis-Herman-Rothery model (GHR, also known as GM models), offer one possibility of explanation of how a vehicle choose its acceleration according to leader's position, velocity and acceleration [5]. Since established, the model was calibrated by several investigations (spanning at least 30 years [7]) using macro- /micro-scopic data. This modelassumes the follower's acceleration depend on its speed, the spacing and relative speed between leader and follower, as described in its formulation:

$$
\ddot{x}_{n+1}(t+T)=\frac{\alpha \dot{x}_{n+1}^{m}(t+T)\left[\dot{x}_{n}(t)-\dot{x}_{n+1}(t)\right]}{\left[x_{n}(t)-x_{n+1}(t)\right]^{1}},
$$

where $\mathrm{n}$ indicate leader vehicle and $\mathrm{n}+1$ for follower, $\mathrm{T}$ is delay of response for follower, $\mathrm{x}$ is position of front of vehicle, a, $1, \mathrm{~m}$ are parameters describing the sensitive of self-speed and spacing, and these parameters have no physical explanation. The model was calibrated in various scenarios around world and the result separated seriously [7].

Based on the consideration of complexity of vehicle motion in congestion, we choose another model that describe traffic dynamic in congestion from the viewpoint of individual vehicle: intelligent driver model (IDM) [6]. As stated in previous chapter, this model is capable to reproduce various state and stability of congestion using corresponding real traffic data. Identical case of IDM for vehicle a is expressed as:

$$
\dot{\mathrm{V}}_{\alpha}=\mathrm{a}\left[1-\left(\frac{\mathrm{v}_{\alpha}}{\mathrm{v}_{0}}\right)^{\delta}-\left(\frac{\mathrm{s}_{*}}{\mathrm{~s}_{\alpha}}\right)^{2}\right], \mathrm{s}_{*}=\mathrm{s}_{0}+\mathrm{Tv}+\frac{\mathrm{v} \times \Delta \mathrm{v}}{2 \sqrt{\mathrm{ab}}},
$$

where $\mathrm{v}$ means velocity and $\Delta \mathrm{v}$ is relative velocity, $\mathrm{s}$ means bumper to bumper spacing, $\delta$ is acceleration exponent and recommends as $4, \mathrm{~s}_{0}$ is jam distance and recommends as $2 \mathrm{~m}, \mathrm{v}_{0}$ is desired velocity and recommends as $120 \mathrm{~km} / \mathrm{h}$, T is safe time headway and recommends as $1.6 \mathrm{~s}$, a is maximum acceleration, $\mathrm{b}$ is desired deceleration. Among these parameters, a, b and $\mathrm{v}_{0}$, as stated in their thesis, mostly affect the shape of graph of the function (most type of congestion was reproduced by only adjust parameter $b$ in their research). All these recommended values were calibrated by traffic data in German freeways in [6].

In addition, Newell's simplified car-following, which containing only two parameter for each individual vehicle, is used to analyze driving characteristics and its interplay with various empirical findings such as anticipation phenomenon and stop-and-go waves [8, 9]. However, these phenomenon is expressed as time-evolution of the two parameters in graph in the two investigation, which indicate instability of the parameters and may undermine accuracy in prediction process. 


\section{Trajectory prediction based on iteration method}

The primarily objective of trajectory prediction is to estimate collision probability. Basically a potential collision in car-follow scenario (rear-end collision) is estimated as Time To Collision (TTC), and warning is usual triggered when TTC is below specific threshold: $2.6 \mathrm{~s}-4 \mathrm{~s}$ for most existing or published collision warning systems according to overview in [4].

Such a system assume constant velocity of two vehicles and TTC can thus be calculated by spacing divided by relative speed of the two vehicles. However, this assumption is, as claimed by Horowitz and Dingus that only $25 \%$ rear-end collision can regard as continuous car-following state [10], may lead to misestimation. Furthermore, the dynamic of leader and self-vehicle is not the only factor that affecting the result motion of a vehicle - one may adjust the motion depending on the anticipation of future trend of surrounding vehicles. As a result, possibility of misestimate of future trajectories for both ego and leading vehicle may raise, leading to false alarms.

\section{Iteration method.}

In order to simulate the inconstant property of leader and estimate its influence on follower's trajectory, we assume that: all drivers can change their state during the predicted period. That is, once a vehicle $\mathrm{n}$ change its speed and acceleration according to its leader vehicle $n-1$, the state change of vehicle $n$ will also influence its follower vehicle $n+1$ when the change is captured by $n+1$. Meanwhile, based on the assumption, each driver can pre-estimate the future trend of surrounding traffic and choose a proper acceleration during the predicted period as its reaction accordingly. In conclusion, trajectory prediction using iteration method can be expressed as:

$$
\mathrm{x}_{\mathrm{n}}(t+T)=\sum_{i=1}^{T / \tau} F\left(\mathrm{x}_{\mathrm{n}}(t+(i-1) \times \tau), \mathrm{x}_{\mathrm{n}-1}(t+(i-1) \times \tau), \tau\right),
$$

Where $x_{n}(t)$ indicate the position of vehicle $n$ at time $t$, vehicle $n-1$ is the leader of vehicle $n, \tau$ is period of drivers adjust their motion according to leader, $\mathrm{F}$ is a function of position change of vehicle $\mathrm{n}$ and is obtained by the dependent variables (two vehicles' position and differential respect to time, and $\tau$ ). Thus $\mathrm{F}$ can be a functional transformation of car-following models whose dependent variable is position and velocity of the two vehicles, and one can obtain predicted position using Eq. 1 or Eq.2, or other proper car-following models. Besides, we can also expand the function and change the variable to position (and differential respect to time) of vehicle $n, n-1, n-2, \ldots, n-(T / \tau)+1$. Meanwhile, the count of vehicles that affect the motion of ego vehicle $n$, is determined by $\mathrm{T}$ and $\tau$, that is, we can use this lever to adjust the trajectory prediction model from normal car-following model to extremely cooperation mode and rapidly transit to equilibrium state (if the parameter make the model stable and any disturbance will be damped in the model).

\section{Parameter calibration.}

We use the trajectory information in NGSIM data, a dataset record all vehicles' positional information of on a section of a highway, to calibrate the driving characteristics of drivers whose motion during the recorded period can regarded as car-following state. According to statement in Highway Capacity Manual 2010 and 2000 that car-following state is the cars following the leader with a time headway no more than 3s [13], and respect to total congested state (time headway in this state usually over 3s), we select a set of cars in NGSIM data who perform constant car-following state. In addition, in order to dilute the influence of lane-changing and merging, we select the vehicles in the two inner lane with no lane-changing, and car-following state maintain at least 30s. 209 pairs of leader and follower is selected based on these condition.

Several investigation object to testify the accuracy of NGSIM data [11, 12], and indicate relatively lower accuracy of velocity and acceleration comparing to accuracy of position. Hence we calibrate the parameters based on the condition that calibrated model have lowest total absolute value of position deviation in every recorded time interval. We calibrate a, m, 1 in Eq.1 (GM model) and a, b, $v_{0}$ in Eq.2 (IDM model) respectively, then an example to trajectory in delineated in Fig 1 and predict error statistics is shown in Table 
1. Calibrated parameter is synodic with empirical study [7] except parameter 1 in IDM model: it express maximum acceleration, and in empirical study it should between 0.5-1.5. It may due to the recommend parameters (see Eq.2) did not fit the situation of NGSim.

Table 1. Statist of calibrated two model and corresponding prediction deviation. Parameters in GM are not physical character, and the three parameters in IDM express maximum acceleration, desired deceleration and desired velocity, respectively.

\begin{tabular}{c|ccc|ccc|cccc}
\hline \multirow{2}{*}{ Model } & \multicolumn{3}{|c|}{\begin{tabular}{c} 
Mean Parameter \\
\cline { 2 - 10 }
\end{tabular}} & \multicolumn{2}{|c|}{ l, m for GM and a, b, $\mathrm{v}_{0}$ for IDM) } & \multicolumn{2}{c|}{$\begin{array}{c}\text { Deviation } \\
\text { without iteration[m] }\end{array}$} & \multicolumn{3}{c}{$\begin{array}{c}\text { Deviation } \\
\text { with iteration[m] }\end{array}$} \\
\hline GM & 1.026 & 0.831 & 0.621 & 0.327 & 1.471 & 20.03 & 0.136 & 1.346 & 26.33 \\
\hline IDM & 0.108 & 1.714 & 23.84 & 0.972 & 2.818 & 19.87 & 0.992 & 2.735 & 19.60 \\
\hline
\end{tabular}

\section{Accuracy of trajectory prediction}

As most common threshold of warning is TTC $<3 \mathrm{~s}$, the efficiency of proposed trajectory prediction method is assessed by predicting the position of $3 \mathrm{~s}$ later for each interval with/without iteration method, respectively (that is, the accuracy of the model is determined by deviation between predicted position and actual position). In addition, we also compare the calibrated two car-following model with/without iteration because of the different transition form ofF in Eq. 3 in iteration model. In the case of no iteration, deviation of calibrated car-following model is equal to the deviation obtained in calibrate process; it is because that they have same predicting period and parameter for each drivers. In the case of iteration, follower are assumed to change their motion (acceleration, in the two car-following model) state each 1s of predict period and maintain the motion in the interval, meanwhile, $\tau=1 \mathrm{~s}$ in Eq.3. Predict error statistics of result is shown in Table 1, and an example of predicted positional deviation during a car-following scenario is delineated in Fig.1.

When comparing to both of statist aspect and example trajectory, one can easily find that: when the car-following model is chose properly, the proposed trajectory prediction model based on iteration will dilute the deviation and reduce both arithmetic average and absolute value of deviation in most cases. We also find that the iteration method has little effect on IDM, while it is huge on GM. It may be caused by the complexity of the model (it contain 7 parameters and most of them has physical meaning).

Besides, deviation for GM modelalso show some intrinsic communication with leader's motion: the model inclined to overestimate the acceleration when the leader is decelerating and underestimate the acceleration when the leader is accelerating. In other constant car-following situation, the performance of trajectory prediction model and iteration method is optimistic.

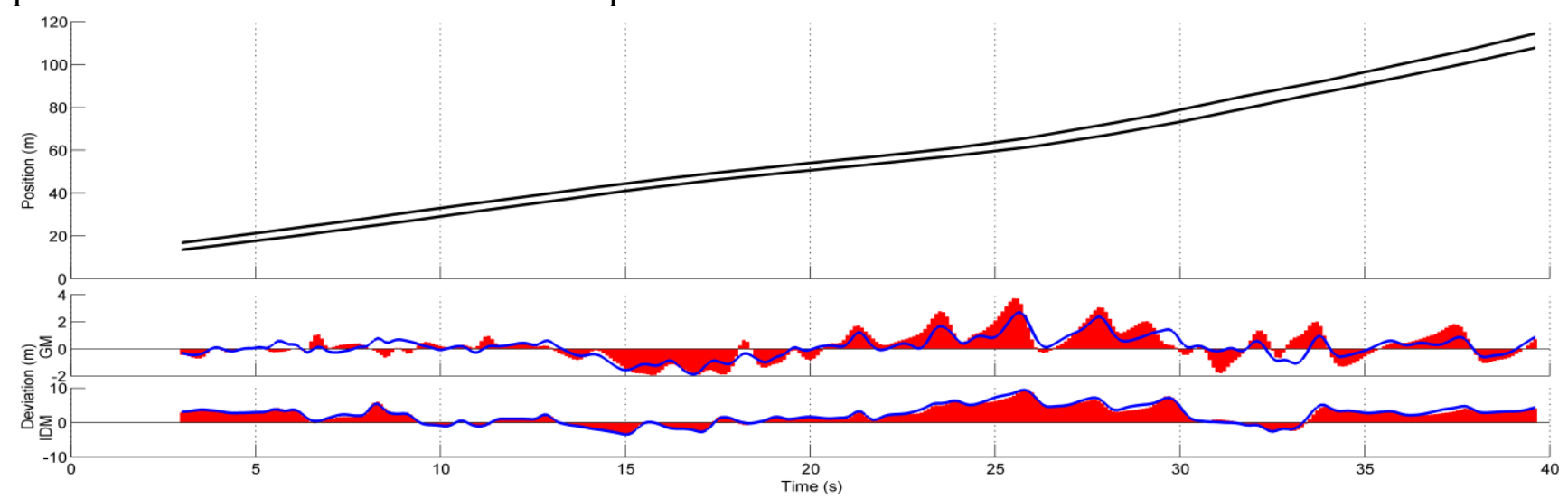

Fig.1 Trajectory of vehicle 179 and accuracy of trajectory prediction for GM/IDM model. In first graph, vehicle 179's leader's trajectory is depicted as upper line while 179 is the other line. In the last two graph, Deviation of without/with iteration is delineated as red aero, blue line, respectively. 


\section{Conclusion and future work}

In the paper, a vehicle trajectory prediction model is proposed and testified. The model utilized the capability of car-following models that they can reproduce various driving behavior and traffic phenomenon, and result shown that a well calibrated car-following model can help to predict the future trajectory of vehicles, which will increase the accuracy of collision warning systems. Besides, the experiment result of iteration method in Fig. 1 and Table 1 also shed light on the anticipation property of drivers that they may change their motion according to anticipation of future trend of surrounding traffic.

While the accuracy of the model is optimistic in this case, other extrinsic factors may be responsible that constrain its utility in collision warning system: firstly, drivers are affected by various factors such as lane-changing vehicle and heterogeneous of traffic (the appearance of tracks and motorcycle). Secondly, in real traffic, the positional information of surrounding vehicles are hard to obtain, and accuracy of trajectory prediction is undermined by instability of vehicular network and error of GPS information. Thus, the primarily objective of future research direction on this is to estimate the influence of these factors and improve the applicability of the proposed trajectory prediction model.

\section{References}

[1] S. Ammoun, F. Nashashibi, Design and efficiency measurement of cooperative driver assistance system based on wireless communication devices. Transportation research part C: emerging technologies, 18(2010) 408-428.

[2] Information on http://www-nrd.nhtsa.dot.gov/Pubs/13WPPP.pdf.

[3] J.A. Misener, R. Sengupta, H. Krishnan, Cooperative collision warning: Enabling crash avoidance with wireless technology. 12th World Congress on ITS. (2005) 6-10.

[4] A. Mehmood, Development of framework for in-vehicle rear-end collision warning system considering driver characteristics, Doctoral Dissertation, Ryerson University, Toronto, 2010.

[5] D.C. Gazis, R. Herman, R.W. Rothery, Nonlinear Follow-The-Leader Models of Traffic Flow, Operations Research, 9(1961) 545-567.

[6] M. Treiber, A. Hennecke, D. Helbing, Congested traffic states in empirical observations and microscopic simulations, Physical review E, 62(2000) 1805-1824.

[7] M. Brackstone, M. McDonald, Car-following: a historical review, Transportation Research Part F 2(1999) 181-196.

[8] D. Chen, J.A. Laval, S. Ahn, Microscopic traffic hysteresis in traffic oscillations: A behavioral perspective, Transportation Research Part B, 46(2012) 1440-1453.

[9] Z. Zheng, S. Ahn, D. Chen, The effects of lane-changing on the immediate follower: Anticipation, relaxation, and change in driver characteristics, Transportation Research Part C, 26(2013) 367-379.

[10] A.D. Horowitz, T.A. Dingus, Warning Signal Design: A key human factors issue in an in-vehicle front-to-rear-end collision warning system, Proceedings from the Human Factors and Ergonomics Society 36th Annual Meeting, Atlanta, (1992) 1011-1013.

[11] C. Thiemann1,M. Treiber, A. Kesting, Estimating acceleration and lane-changing dynamics based on NGSIM trajectory data, Transportation Research Board, 44(2008) 90-101.

[12] V. Punzo, M.T. Borzacchiello, B. Ciuffo, On the assessment of vehicle trajectory data accuracy and application to the Next Generation SIMulation (NGSIM) program data, Transportation Research Part C, 19(2011) 1243-1262. 
[13] M.R. Morris, N.J. Pedersen, R.E. Skinner, Highway Capability Manual 2010, fifth ed. Washington DC, 2010. 